\title{
Effect of HIP parameters on the micro-structural evolution of a single crystal Ni-based superalloy
}

\author{
Inmaculada Lopez-Galilea ${ }^{1, \mathrm{a}}$, Stephan Huth ${ }^{1, \mathrm{~b}}$, Marion Bartsch ${ }^{2, \mathrm{c}}$, \\ Werner Theisen ${ }^{1, d}$ \\ ${ }^{1}$ Ruhr-Universität Bochum, Lehrstuhl Werkstofftechnik, 44780 Bochum, Germany \\ ${ }^{2}$ Deutsches Zentrum für Luft- und Raumfahrt (DLR), Köln-Porz, Germany \\ alopez@wtech.rub.de, ${ }^{\text {}}$ huth@wtech.rub.de, ${ }^{\text {}}$ marion.bartsch@dlr.de, ${ }^{d}$ theisen@wtech.rub.de
}

\begin{abstract}
Key words: Hot Isostatic Pressing (HIP), Diffusion, Single Crystal, Microstructure, Ni-based superalloys.

Abstract. For reducing the porosity of single crystal (SX) nickel-based superalloys, Hot Isostatic Pressing (HIP) is used. High pressures of about 100-170 MPa lead to local deformation, which close the pores. However, since HIP also requires high temperatures $\left(1000-1200^{\circ} \mathrm{C}\right)$ it has a pronounced effect on the microstructure and the local distribution of elements. This contribution analyses the effect of different HIP treatments on both the microstructure and the segregation of the SX superalloy LEK94 in the as-precipitation-hardened state. In addition, the effects of rapid or slow cooling are analyzed. To distinguish the effect of pressure from those of temperature, the HIPed samples are compared with specimens annealed at atmospheric pressure.
\end{abstract}

\section{Introduction}

Second-generation of SX nickel-based superalloys exhibit a combination of excellent strength, good creep properties and fatigue resistance at high temperatures. These materials are currently used as blades in gas turbine and aircraft engines. They owe their properties to the large amount ( $\sim 70$ vol.\% [1]) of finely dispersed precipitates of the ordered $\left(\mathrm{L1}_{2}\right) \gamma^{\prime}$ phase, which show a very high solvus temperature $\left(\sim 1200^{\circ} \mathrm{C}\right)$.

One of the main drawbacks of Ni-based superalloys is the lack of strength and ductility due to the formation of microporosity during casting, which also results in the scatter of the mechanical properties of cast components [2]. Furthermore, the shrinkage cavities can act as crack initiation sites and promote crack propagation, leading to premature rupture of the components. Therefore, it is an important issue to reduce the microporosity generated during casting.

In order to solve this task and to obtain superior mechanical behaviours, the application of HIP on the material reduces the size and amount of porosity [3] via a combination of plastic deformation, creep and diffusion bonding [4-6]. Simultaneously, the alloy composition is homogenized to some extent since the HIP partially dissolves $\gamma^{\prime}$ [7-9]. There have been attempts to apply HIP both directly after casting and after precipitation hardening.

In the present work, the HIP technology was applied to the SX Ni-based superalloy LEK94 to investigate the evolution of the microstructure - mainly the homogenization of the alloy composition and the morphology of the $\gamma^{\prime}$ precipitates in the dendrite core - after HIP by using scanning electron microscopy. One aim of the study is to determine the relationship between the HIP parameters, mainly pressure and temperature, with the observed microstructure.

\section{Experimental procedure}

The SX Ni-based superalloy LEK94 has been investigated in this work. This material was provided and developed by MTU Aero Engines (München, Germany) in the ready to use state, meaning it has been subjected to both a homogenization/solution annealing and a precipitation hardening treatment after casting.

Small specimens $\left(5 \times 5 \times 8 \mathrm{~cm}^{3}\right)$ were heat treated at $1180^{\circ} \mathrm{C}$ at either ambient pressure or $170 \mathrm{MPa}$, as shown in Table 1, in order to study the effect of pressure on the evolution of the microstructure and on the segregation. 
Low pressure (LP) annealing tests were performed in laboratory furnaces in an Argon gas atmosphere to avoid oxidation. Samples were introduced into the pre-heated furnace and after a holding time of 4 hours they were either quenched in water (LP-F), or cooled slowly by switching off the furnace, which leads to a very long cooling time of about 10 hours (LP-S).

High pressure (HP) treatments were carried out in two different hot isostatic presses. The first one (HP-S) was carried out in a laboratory HIP unit without accelerated cooling that lead to a cooling time of about 4 hours. The other specimen (HP-F) was treated in an industrial HIP furnace with the possibility of fast cooling. Although the pressure was progressively released during a cooling time of around 1 hour, after 15 min cooling the sample reached the temperature of $800^{\circ} \mathrm{C}$, below which not major microstructural changes are expected.

Each specimen was analyzed in terms of the morphological change of $\gamma^{\prime}$ phase using a scanning electron microscope (SEM) equipped with an energy dispersive X-ray diffraction (EDX) unit. For the analysis, the specimens were cut perpendicularly to the solidification direction of the cast process, $\langle 100>$. All the specimens were ground and mechanically polished. For the EDX analyses an accelerated voltage of $8 \mathrm{keV}$ was used, which implies an interactive spatial resolution of approximately $150-200 \mathrm{~nm}$.

\section{Results and discussion}

Microscopic changes in the size and morphology of $\gamma^{\prime}$ precipitates in the dendrite core depending on the treatments were addressed via SEM images with high magnifications, up to 30000 . Figure 1 shows the typical $\gamma / \gamma^{\prime}$ structure developed in a precipitation hardening treatment. This is the starting microstructure of the specimens subjected to different treatments. Figure 4 and Figure 5 show the dendrite core microstructures developed after the different heat treatments.

Table 1. Studied samples and heat treatments. Figure 1. Cross-sectional SEM image of The letter $P$ stands for pressure, $T$ for LEK94 in a dendrite core after Precipitation temperature and t for time.

\begin{tabular}{|c|c|c|c|c|}
\hline Specimens & $\mathbf{P}[\mathbf{M P a}]$ & $\mathbf{T}\left[{ }^{\circ} \mathbf{C}\right]$ & $\mathbf{t}[\mathbf{h}]$ & cooling \\
\hline LEK94 & - & - & - & - \\
\hline LP-F & 0.1 & \multirow{4}{*}{1180} & 4 & Fast \\
\hline LP-S & 0.1 & & 4 & Slow \\
\hline HP-F & 170 & & 10 & Fast \\
\hline HP-S & 170 & & 4 & Slow \\
\hline
\end{tabular}

\section{Hardening.}

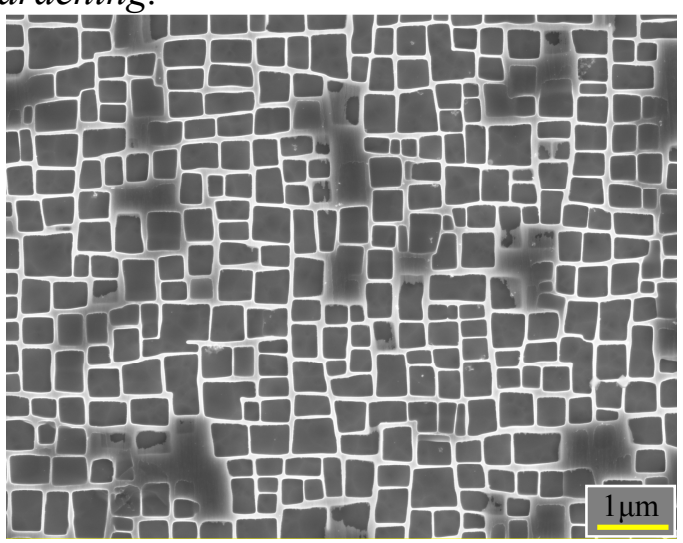

Figure 2. Concentration of the main alloy elements after precipitation hardening as well as after different treatments in the core (left) and the interdendritic region (right). Chemical composition in [wt.\%] was measured using EDX.

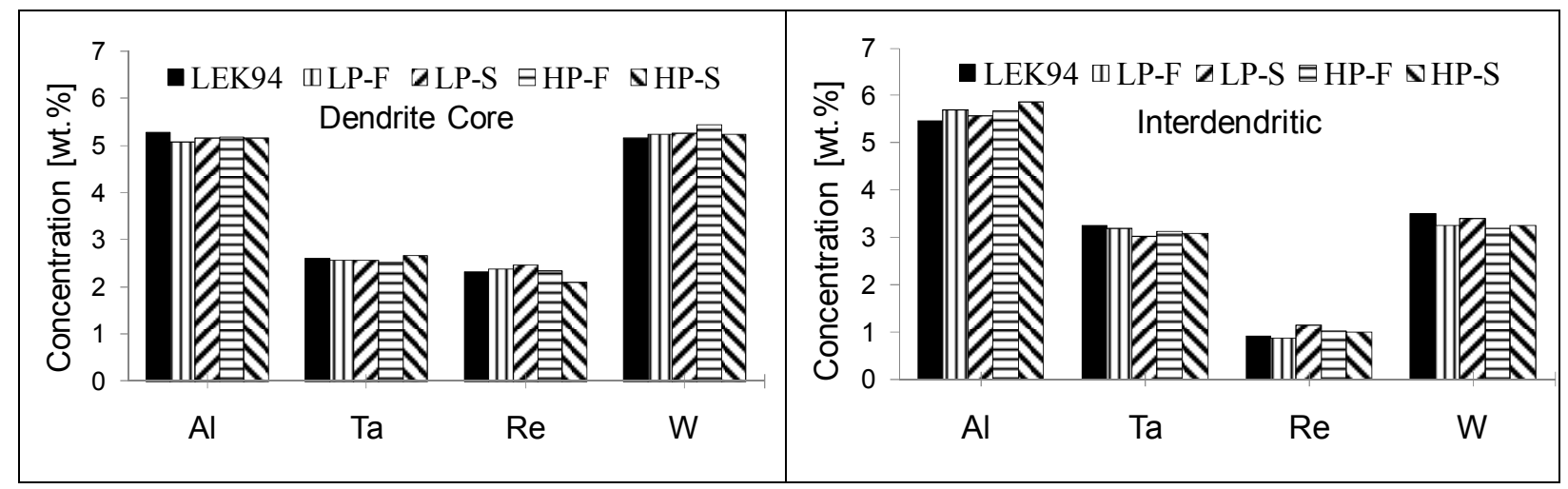


Figure 3. Concentration at the dendrite core of the main alloy elements after precipitation hardening as well as after different treatments in the $\gamma$ matrix (left) and in the $\gamma^{\prime}$ (right). Chemical composition in [wt.\%] was measured using EDX.

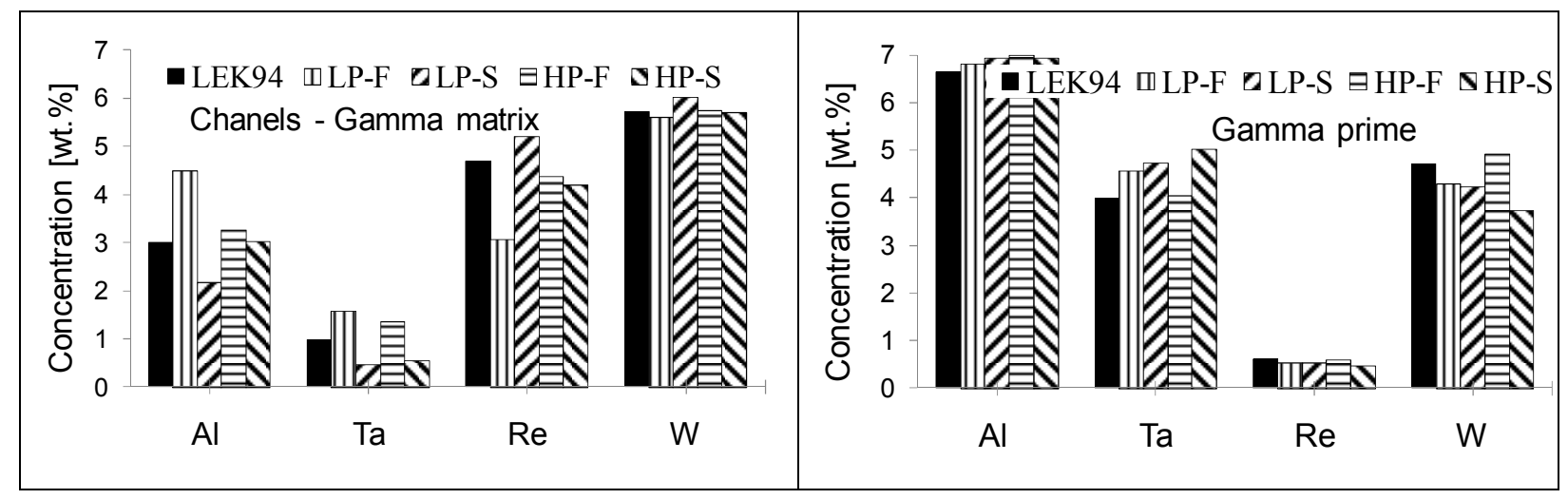

During solidification, microsegregation, which is caused by different solubilities of the alloying elements in the growing solid phases and the melt, leads to chemical inhomogeneities of the material on the length scale of the primary dendrite spacing $(200-400 \mu \mathrm{m})$. Although this segregation is reduced during the solution annealing, it still remains in the material that is ready to be used. In this way, the elements $\mathrm{Al}$, Ti and $\mathrm{Ta}$ are mainly present in the $\gamma^{\prime}$ phase and their concentrations are higher in the interdendritic area because these elements enrich in the melt during casting, while $\mathrm{W}, \mathrm{Re}$ and Mo enrich in the $\gamma$ gamma matrix and their concentrations are higher in the dendrite core than in the interdendritic areas, because they deplete in the melt. Therefore, the composition of the dendrite changes from the dendrite core towards the interdendritic regions. Figure 2 shows the concentration profiles, for the main elements in the core (Figure 2-left) and in the interdendritic zone (Figure 2-rigth) for the material in the initial stage as well as for the other treated specimens. These values are averages of several measurements. In general, from the concentration profile shown in Figure 2, in can be concluded that the additional heat treatments after precipitation hardening did not significantly modify the global composition of the dendrites. Slight fluctuations can be observed in the interdendritic regions, without remarkable trends. Thus, they could be considered within experimental error, because due to the irregular morphology developed in these areas, it was not always possible to measure the composition in a similar interdendritic region for all samples.

Figure 3 shows the concentration profiles of major elements in phase $\gamma$ (Figure 3-left) and $\gamma^{\prime}$ (Figure 3-rigth) in the dendrite core, for the material in the initial stage as well as for the other treated specimens. These values are averages of several measurements. The concentration values indicated for the $\gamma$ phase in Figure 3-left refer to the values measured in the channels. So, as in some samples small particles of $\gamma^{\prime}$ appear in these channels, the indicated composition for the $\gamma$ matrix means the one measured in the channel that contains $\gamma$ and small $\gamma^{\prime}$ particles. 
Figure 4. Cross-sectional SEM images of the dendrite cores after the different heat treatments at low pressure with a subsequent fast cooling a) LP-F or slow cooling b) LP-S.

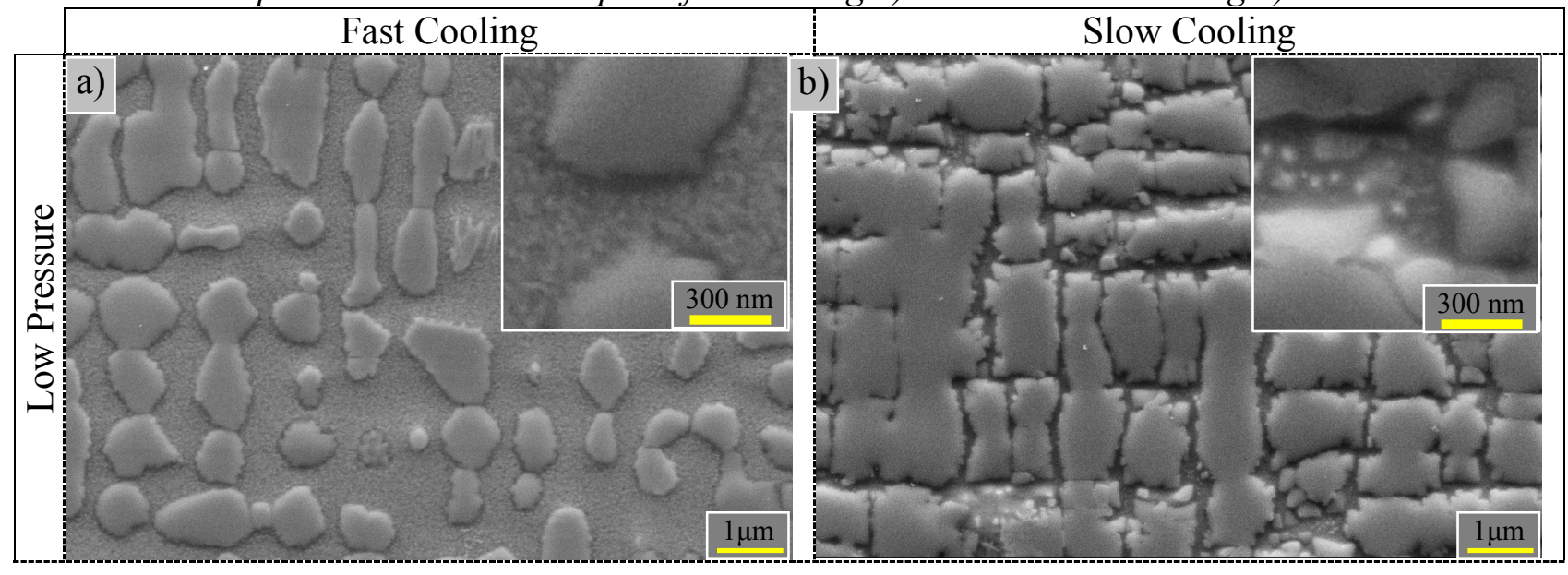

Figure 4 shows the cross-sectional SEM images of the resulting $\gamma / \gamma^{\prime}$ morphology in the dendrite core after the heat treatment at low pressure with a subsequent fast cooling a) LP-F or slow cooling b) LP-S.

LP-F (Figure 4-a): The shape of the primary $\gamma^{\prime}$ particles in the dendrite core is drastically changed from cuboidal to round. This kind of change of shape is typical for Ni-based superalloys used for turbine blades after exposure at high temperature for a long time [10]. In addition, the total amount of $\gamma^{\prime}$ is considerably reduced because at these high temperatures, the equilibrium amount of $\gamma^{\prime}$ phase is lower, resulting in partial dissolution of $\gamma^{\prime}$ precipitates. The size of the single precipitates however is increased significantly. When cooling down rapidly, very fine secondary $\gamma^{\prime}$ particles precipitate in the $\gamma$ matrix between the larger round primary $\gamma^{\prime}$ particles. The dissolution of the primary $\gamma^{\prime}$ phase implies that the composition of the channels consisting of $\gamma$ phase and tiny secondary $\gamma^{\prime}$ particles in the dendrite core is enriched in $\gamma^{\prime}$ formers, as $\mathrm{Al}$ and Ta, while it is reduced in $\gamma$ formers, mainly $\mathrm{W}$ and Re.

LP-S (Figure 4-b): The slow cooling results in growth and coalescence of primary and secondary $\gamma^{\prime}$ precipitates at the expense of some of the very fine $\gamma^{\prime}$ precipitates and finally the formation of fishbone shapes $[9,11,12]$. These complicated morphological changes suggest that the initial $\gamma^{\prime}$ precipitates had undergone a partial solution during holding time at $1180^{\circ} \mathrm{C}$, showing the morphology of primary $\gamma^{\prime}$ such as found for LP-F. The growth and coalescence of the very fine secondary $\gamma^{\prime}$ precipitates during slow cooling results in a change in the composition of the matrix phase, decreasing the amount of $\gamma^{\prime}$ formers, $\mathrm{Al}$ and Ta and increasing the $\gamma$ formers concentration.

Figure 5 shows the cross-sectional SEM images of the resulting $\gamma / \gamma^{\prime}$ morphology in the dendrite core after the heat treatment at high pressure with a subsequent fast cooling a) HP-F or slow cooling b) HP-S.

HP-F (Figure 5-a): The microstructure developed for the specimen HP-F is not very different from

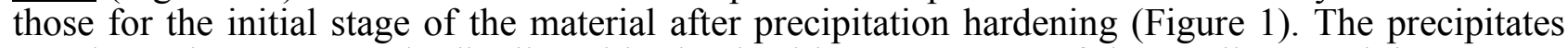
are almost homogeneously distributed in the dendrite core. Some of the small $\gamma^{\prime}$ precipitates seem to have dissolved because some relatively big areas of $\gamma$ matrix appeared. In these $\gamma$ matrix areas and also in the channels between the primary $\gamma^{\prime}$ precipitates, very small secondary $\gamma^{\prime}$ particles were precipitated during fast cooling. The matrix composition slightly increases in $\gamma^{\prime}$ formers, but remarkably less than in the LP-F sample. These observations can be explained by a retarded diffusion coefficient of solute elements under high pressure [9].

HP-S (Figure 5-b): As it was described for the specimen LP-S, some small $\gamma^{\prime}$ precipitates seem to have dissolved and re-precipitated in the neighbourhood of the primary precipitates. Similarly to LP-S, the slow cooling results in growth and coalescence of primary and secondary $\gamma^{\prime}$ precipitates on the expense of very fine $\gamma^{\prime}$ precipitates. The growth and coalescence are slightly less expressed compared to the LP-S specimen, since the diffusion coefficient of solute under high pressure is lower than that under atmospheric or low pressure [9]. Compared to the morphological changes of $\gamma^{\prime}$ precipitates, the differences in composition shown for the $\gamma$ matrix are less severe than those obtained for the specimen LP-S, because the amount of $\gamma^{\prime}$ phase dissolved and re-precipitated was small. 
Figure 5. Cross-sectional SEM images of the dendrite cores after the different heat treatments: a) $H P-F$, b) $H P-S$.

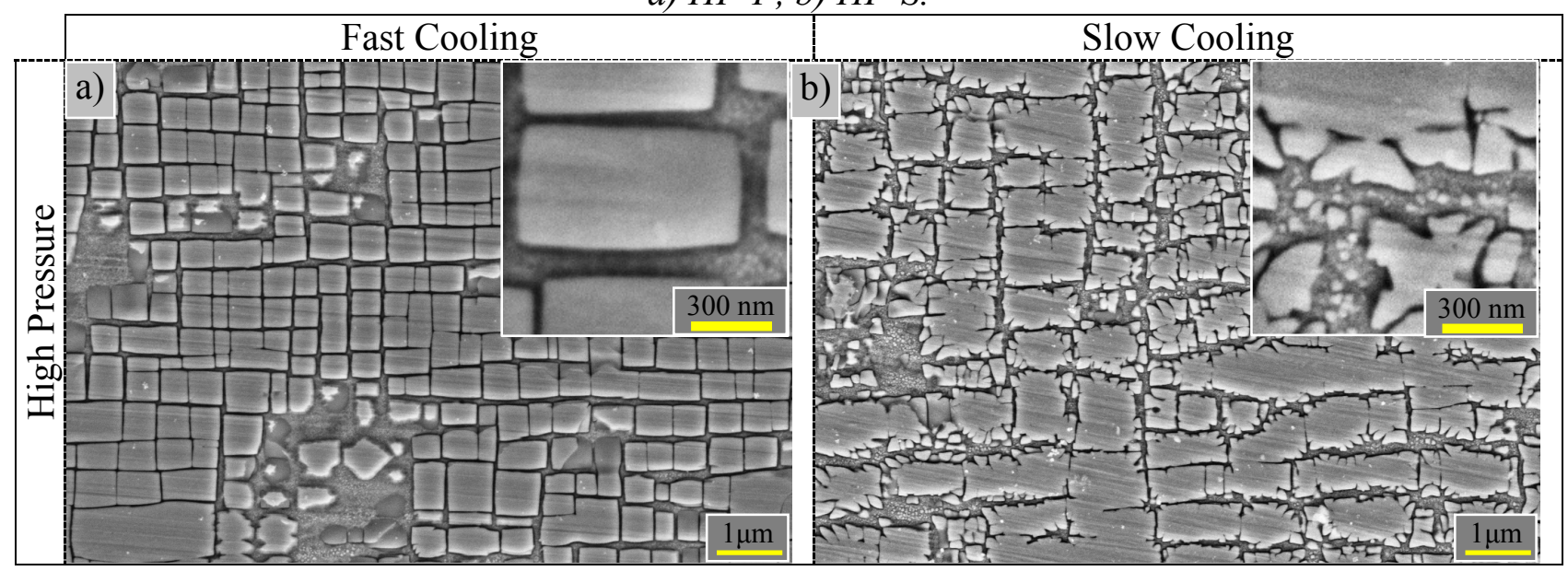

\section{Summary and conclusions}

In the present study, small samples of the LEK94 single-crystal superalloy have been used in order to study microstructure changes due to thermal treatment and isostatic pressure. The samples underwent different heat treatments at $1180^{\circ} \mathrm{C}$ : HIP tests under $170 \mathrm{MPa}$ and annealing at atmospheric pressure. The investigation led to the following conclusions:

1. Annealing at $1180^{\circ} \mathrm{C}$ under atmospheric pressure changes the shape of $\gamma^{\prime}$ precipitates from cuboidal to round. Simultaneously, the volume fraction of primary $\gamma^{\prime}$ is drastically reduced, due to the higher solubility of this phase at high temperatures. On fast cooling, the same primary $\gamma^{\prime}$ microstructure was maintained while in the matrix very small secondary $\gamma^{\prime}$ particles were formed. The composition measured in the matrix after fast cooling reveals its enrichment in $\gamma^{\prime}$ formers and impoverishment in $\gamma$ formers, due to the initial dissolution of primary $\gamma^{\prime}$ phase, which re-precipitated in the matrix as very fine secondary $\gamma^{\prime}$ phase.

2. High isostatic pressure is associated with several microstructural processes, including retarded diffusion of the alloying elements. In this way, the morphology of the primary $\gamma^{\prime}$ phase after the HIP treatment and fast cooling is not significantly different from that of the initial LEK94 material. However, small $\gamma^{\prime}$ precipitates in the $\gamma$ channels are observed due to the fast cooling. As a consequence of the low diffusion coefficient under high pressure, the matrix composition only slightly increases in $\gamma^{\prime}$ formers.

3. The slow cooling rate either after annealing at atmospheric pressure or after HIP, leads to massive growth and coalescence of secondary $\gamma^{\prime}$ precipitates around the original primary $\gamma^{\prime}$ particles, from which the $\gamma^{\prime}$ former elements were dissolved during the heat treatment. This degradation of the microstructure by a coarsening process, changing the final shape of the particles, will reduce the excellent mechanical properties normally shown by LEK94. Thus, HIP should be performed prior to homogenization instead afterwards, if fast cooling from HIP-temperature cannot be accomplished.

4. A detailed control of the cooling is necessary in order to control the final morphology and amount of the $\gamma^{\prime}$ precipitates. The differences observed on the HIPed specimens are probably not due to holding time ( $4 \mathrm{~h}$ and $10 \mathrm{~h}$ ) but to the cooling ratio. As a consequence, the correct selection of the cooling speed could allow us to obtain the desired microstructure. 


\section{Acknowledgment}

This work was supported by the state of North Rhine-Westphalia through the Research Department "Integrity of Small-Scale Systems /High-Temperature Materials" (RZ, GE, AL).

\section{Literature References.}

[1] R.C. Reed. The Superalloys. Cambridge: Cambridge University Press (2006).

[2] B.S. Bokstein, A.I. Epishin, T. Link, V.A. Esin, A.O. Rodin and I.L. Svetlov. Scripta Materialia Vol.57. pp 801-804 (2007).

[3] J.C. Chang, Y.H. Yun, C. Choi and J.C. Kim. Journal of Materials Engineering and Performance. Vol.12(4). pp. 420-425 (2003).

[4] G. Mälzer, R.W. Hayes, T. Mack and G. Eggeler. Metallurgical and Materials Transactions A. Vol. 38A. pp. 314-327 (2007).

[5] P. Wangyao, S. Joypradit, P. Tuengsook, V. Homkrajai, S. Khunthon. Journal of Metals, Materials and Minerals. Vol. 14 (1). pp. 89-94 (2004).

[6] P. Wangyao, G. Lothongkum, V. Krongtong, W. Homkrajai, N. Chuankrerkkul. Chiang Mai Journal Science. Vol. 36(3). pp. 287-295 (2009).

[7] R.J. Simpkins II, M. P. Rourke, T. R. Bieler, P.A. McQuay. Materials Science and Engineering A.Vol.463 (1-2). pp. 208-215 (2007).

[8] H.Y. Bor, C. Hsu, C.N. Wei. Materials Chemistry and Physics. Vol. 84. pp. 284-290 (2004).

[9] M.T. Kim, S.Y. Chang, J.B. Won. Materials Science and Engineering A.Vol.441. pp.126-134 (2006).

[10] J.C. Chang, Y.H. Yun, C. Choi, J.C. Kim. Engineering Failure Analysis. Vol 10. pp. 559-567 (2003).

[11] H.T. Lee, S.W. Lee. Journal of Materials Science Letters. Vol. 9. pp. 516-517 (1990).

[12] B.C. Wilson and G.E. Fuchs. Metallurgical and materials transactions A. Vol 41A. pp. 12351245 (2010). 